\title{
Operando Scanning Electron and Microwave Microscopies in Plasmas: A Comparative Analysis
}

Andrei Kolmakov ${ }^{1}$ and Alexander Tselev ${ }^{2}$

${ }^{1}$ National Institute of Standards and Technology, Gaithersburg, Maryland, United States, ${ }^{2}$ Department of Physics \& CICECO-Aveiro Institute of Materials/ University of Aveiro, Aveiro, Aveiro, Portugal

There exists a great need for an operando nanoscale characterization of the evolution of surface compositions and morphologies during plasma-assisted processing. These include sputtering deposition, plasma-assisted etching, enhanced atomic layer deposition relevant for semiconductor and aerospace industries, environmental remediation and treatment of the bio-(medical) interfaces. We have recently proposed using near-field scanning probe-based microwave imaging known as scanning Microwave Impedance Microscopy (sMIM) to image the surfaces immediately (a few seconds) after plasma processing with a sub-100 $\mathrm{nm}$ spatial resolution [1].

In this communication, we report on a true operando sMIM in plasma and its extension to imaging in SEM. The core of our approach is a microflow DC micro-discharge chamber equipped with a few-10s nm-thick SiN membrane transparent to a few $\mathrm{keV}$ electrons and microwave radiation (Figure 1a). This membrane isolates the sMIM probe and SEM column from the plasma environment and thus enables the real-time imaging of the surface of interest under plasma conditions. Using model systems, such as graphene, PMMA films, and polystyrene microparticles, we comparatively explored the performance of the SEM and sMIM in the same plasma-assisted process. In particular, sensitivity, frame rate, spatial resolution, probing depth, and probe-induced effects were evaluated and compared. Figure 1b shows the process of etching of folded graphene by air DC plasma. The same process is depicted in the SEM snapshots (Figure 1c). The sMIM contrast in Figure $1 \mathrm{~b}$ is due to graphene conductivity which degrades significantly after a few seconds of ca. $200 \mathrm{~mW}$ plasma treatment. Under optimal conditions, a resolution of ca. $50 \mathrm{~nm}$ can be achieved for metallic objects with commercially available probes. The contrast formation in SEM is due to local variations in the electron emission yield of the sample. This, in turn, can be affected by the presence of the static charges in the SiN membrane. The spatial resolution can be as high as $10 \mathrm{~nm}$ with the frame rate on the order of $1 \mathrm{~Hz}$. Interestingly, both the microscopies revealed the formation of highly conductive filamentary structures of graphene during plasma etching-the phenomenon that requires further studies. 


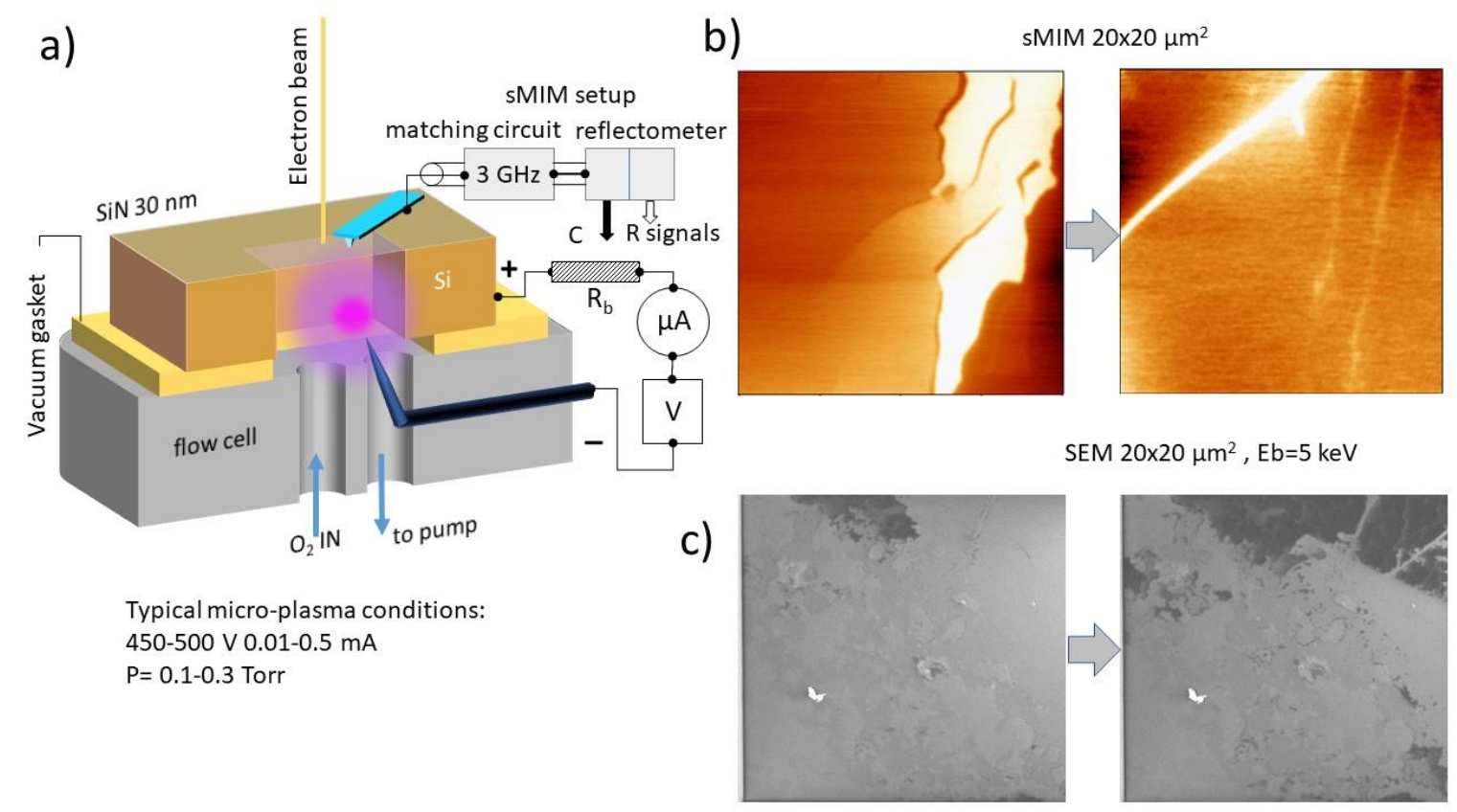

Figure 1. (a). Experimental setup for operando sMIM and SEM studies under DC plasma; (b) sMIM (conductance channel) $20 \times 20 \mu \mathrm{m} 2$ image of the graphene sample before (left panel) and after a few seconds (right panel) of application of DC air plasma ( 0.25 Torr, $500 \mathrm{~V}, 0.4 \mathrm{~mA})$. The overall signal intensity drops (color scales are different in the images) due to local graphene etching, and filamentary structures are formed; (c) A similar sequence of 20x20 $\mu \mathrm{m} 2$ images in SEM (5 keV) taken during DC (0.25 Torr, $450 \mathrm{~V} 0.2 \mathrm{~mA}$ ) plasma treatment

\section{References}

[1] A. Tselev, J. Fagan, and A. Kolmakov, "In-situ near-field probe microscopy of plasma processing," Applied Physics Letters, vol. 113, no. 26, p. 263101, 2018. 